\title{
Estudio comparativo de psicopatología en hijos de padres con trastorno bipolar
}

\author{
Patricia Zavaleta-Ramírez, ${ }^{1}$ Francisco R. de la Peña Olvera, ${ }^{2}$ Manuel Alejandro Vargas-Soberanis, ${ }^{3}$ \\ Miriam Feria Aranda, ${ }^{3}$ Liz Sosa Mora ${ }^{3}$ Adriana Arias Caballero, ${ }^{3}$ \\ Claudia Becerra Palars, ${ }^{4}$ Lino Palacios-Cruz ${ }^{5}$
}

Artículo original

\section{SUMMARY}

Background

The offspring of patients with bipolar disorder (BD) constitute a high risk population for multiple psychiatric disorders that require an early systematic evaluation and longitudinal follow up.

\section{Objective}

To describe and compare the psychopathology profile in children and adolescents who are offsprings of parents with $B D$ versus offspring of non-BD parents from a community sample.

\section{Material and methods}

Parents were evaluated with the Mini International Diagnostic Interview (MINI). Based on the results of the interview, two groups were created: parents with or without BD. Offspring evaluation was carried out with the Kiddie Schedule for Affective Disorders and Schizophrenia for School-age Children Present and Lifetime Version (K-SADS-PL). The functioning was evaluated with the Clinical Global Assessment Scale (C-GAS).

\section{Results}

Ninety-three percent of the bipolar parents' offspring had a long life psychiatric disorder. Externalizing disorders were the most frequent $(81 \%)$. BD parents' offspring showed higher risk for externalizing disorder ( $R M=4.44 ; \mathrm{IC}=95 \% ; 1.43-13.84)$ and for attention-deficit hyperactivity disorder $(\mathrm{RM}=4.44 ; \mathrm{IC}=95 \% ; 1.43-13.84 ; \mathrm{p}=.01)$ and oppositional defiant disorder ( $\mathrm{RM}=3.06$; $\mathrm{IC}=95 \% ; 1.05-8.93)$.

\section{Conclusions}

Bipolar parents' offspring have a higher prevalence for psychiatric disorders, especially in the externalized area. The longitudinal course of early onset psychopathology suggests that this population requires early diagnostic and strategies of treatment to change the trajectories of these disabling chronic disorders.

Key words: Bipolar disorder, offspring, psychopathology.

\section{RESUMEN}

\section{Antecedentes}

Los hijos de padres con diagnóstico de trastorno bipolar constituyen una población de alto riesgo para la presentación de múltiples trastornos psiquiátricos que requieren una evaluación sistemática temprana $\mathrm{y}$ un seguimiento longitudinal.

\section{Objetivo}

Describir y comparar el perfil psicopatológico en hijos de padres con trastorno bipolar versus hijos de padres sin trastorno bipolar en una muestra comunitaria.

\section{Material y métodos}

La evaluación de la psicopatología en los padres se realizó con la Entrevista Mini International Diagnostic Interview (MINI). Con base en los resultados, se crearon los grupos de padres con y sin trastorno bipolar (TBP). Posterior a la misma, se realizó la valoración de psicopatología en sus hijos con el Kiddie Schedule for Affective Disorders and Schizophrenia for School-aged Children Present and Lifetime Version (K-SADS-PL) y el funcionamiento con la Escala de Funcionamiento Global en niños (C-GAS).

\section{Resultados}

El $90 \%$ de los hijos de padres con TBP ha presentado un trastorno psiquiátrico a lo largo de la vida; el grupo de trastornos externalizados fue el más frecuente $(81 \%)$. Los hijos de padres con TBP mostraron mayor riesgo de presentar cualquier trastorno externalizado ( $R M=4.44$; $\mathrm{IC}=95 \%$; 1.43-13.84), mayor riesgo para trastorno por déficit de atención e hiperactividad ( $R M=3.38 ; \mathrm{IC}=95 \% ; 1.18-8.93)$ y trastorno negativista y desafiante $(\mathrm{RM}=3.06 ; \mathrm{IC}=95 \% ; 1.05-8.93)$.

\section{Conclusión}

Los hijos de padres con TBP presentan una alta prevalencia de trastornos psiquiátricos, especialmente en el área de los externalizados. El curso longitudinal de la psicopatología de inicio temprano sugiere que esta población requiere estrategias tempranas de diagnóstico y tratamiento para cambiar las trayectorias discapacitantes de los trastornos crónicos.

Palabras clave: Trastorno bipolar, hijos, psicopatología.

\footnotetext{
Unidad en Hospital, Dirección de Servicios Clínicos, Instituto Nacional de Psiquiatría Ramón de la Fuente Muñiz (INPRFM).

Departamento de Fomento a la Investigación, INPRFM.

Clínica de la Adolescencia, Dirección de Servicios Clínicos, INPRFM.

4 Clínica de Trastornos del Afecto, Dirección de Servicios Clínicos, INPRFM.

Subdirección de Investigaciones Clínicas, INPRFM.
}

Correspondencia: Francisco R. de la Peña, Departamento de Fomento a la Investigación, Dirección de Servicios Clínicos, INPRFM. Calz. México-Xochimilco 101, San Lorenzo Huipulco, Tlalpan, 14370, México, DF. E-mail: adolesc@imp.edu.mx

Recibido: 7 de julio de 2014. Segunda versión: 11 de agosto de 2014. Tercera versión: 10 de octubre de 2014. Aceptado: 13 de octubre de 2014 


\section{INTRODUCCIÓN}

El trastorno bipolar (TBP) es un trastorno del estado de ánimo de curso crónico, fluctuante, con altas tasas de discapacidad, ${ }^{1}$ disfunción, ${ }^{2}$ comorbilidad psiquiátrica ${ }^{3}$ e intentos suicidas, ${ }^{4}$ que genera altos costos en salud para la sociedad. ${ }^{5}$

Los estudios retrospectivos realizados en adultos con TBP han reportado que de $2 \mathrm{O}$ a $30 \%$ de los casos iniciaron antes de los 20 años de edad; ${ }^{6}$ sin embargo, la mayoría de ellos no fueron detectados tempranamente. El retraso en el diagnóstico se atribuye a que antes del inicio de las alteraciones afectivas existen otras manifestaciones de psicopatología en la infancia, como el trastorno disocial (TD) y el trastorno por déficit de atención con hiperactividad (TDAH), que pueden enmascarar los cuadros afectivos y/o de elevación del estado del ánimo. ${ }^{7-9}$

Los hijos de padres con TBP se consideran un grupo de alto riesgo para el desarrollo del mismo trastorno, como lo indican estudios que muestran que la probabilidad de desarrollar este trastorno es del $10 \%$, cuando se tiene un padre afectado, y de $40 \%$, cuando ambos padres lo están. ${ }^{10}$ Asimismo, estudios en gemelos han mostrado que de 70 a $80 \%$ de las manifestaciones clínicas se explican por factores genéticos. ${ }^{11}$

El riesgo que corre esta población no es únicamente el de desarrollar TBP, pues los estudios reportan que $50 \%$ de los hijos de padres con TBP cumplen criterios para al menos un trastorno psiquiátrico, ${ }^{12-15}$ lo que muestra un perfil heterogéneo, pero con mayores tasas de trastornos de conducta disruptiva, trastornos de ansiedad y del sueño, así como trastornos del ánimo, cuando se comparan con los hijos de padres $\sin$ TBP. ${ }^{16-18}$

Es así que, en comparación con la población general, los hijos de padres con TBP corren un riesgo 2.5 veces mayor de desarrollar cualquier trastorno psiquiátrico y cuatro veces mayor probabilidad de presentar un trastorno del estado de ánimo. ${ }^{10}$ Cuando consideramos la perspectiva del desarrollo, los estudios realizados en preescolares reportan una prevalencia ocho veces mayor para la presencia de TDAH, así como mayor frecuencia de síntomas depresivos y maniacos subumbrales, en comparación con los hijos de padres sin TBP. ${ }^{19}$ En la adolescencia se observa un incremento en la prevalencia del diagnóstico de TBP (de 3 a 10\%) y en mayor proporción para cualquier trastorno del ánimo $(40 \%) .{ }^{20}$

El estudio de esta población es interesante debido a las necesidades de atención, disfunción y psicopatología temprana que influyen, en una trayectoria heterotípica, en otros trastornos crónicos y discapacitantes. Este estudio es importante también para diseñar algoritmos de intervención temprana y evaluar su desenlace.

\section{Objetivo}

Describir y comparar la psicopatología en niños y adolescentes hijos de padres con TBP versus hijos de padres sin TBP en una muestra comunitaria.

\section{MATERIAL Y MÉTODOS}

\section{Muestra}

Padres con trastorno bipolar. Se reclutaron por medio de una clínica especializada en el diagnóstico y tratamiento de los trastornos del ánimo de un hospital de psiquiatría de tercer nivel, de octubre de 2009 a julio de 2010. Se invitó a participar a los pacientes con hijos biológicos de seis a 18 años de edad.

Se excluyó a los padres con diagnóstico de TBP secundario a uso de sustancias o causa médica y a aquellos padres que presentaron durante la evaluación algún estado psiquiátrico que les impidiera otorgar su consentimiento informado.

Padres sin TBP de la comunidad. Se reclutaron en una escuela primaria y secundaria particular aledaña a la zona geográfica en que se llevó a cabo el estudio. Se convocó a los padres para recibir un curso informativo sobre trastornos de ansiedad y después del mismo se les invitó a participar en el estudio. Se incluyeron todos los padres que aceptaron participar voluntariamente. Se excluyó a aquellos a quienes durante la evaluación se les diagnosticó TBP y/o que tuvieran familiares conocidos de primer grado con este diagnóstico.

Entrevistas diagnósticas. Para la confirmación y/o el diagnóstico de los padres, se utilizó la Mini International Neuropsychiatric Interview (MINI), adaptada para Centro y Sudamérica. ${ }^{21}$ Ésta es una entrevista diagnóstica estructurada que evalúa los principales trastornos psiquiátricos del Eje I de acuerdo con el DSM-IV y la CIE-10. Tiene una confiabilidad interevaluador de 0.67 a 0.95 para las distintas categorías diagnósticas. Fue aplicada por un evaluador independiente a las evaluaciones de los hijos.

Para evaluar la psicopatología en los menores, se utilizó el Kiddie Schedule for Affective Disorders and Schizophrenia for School-Age Children Present and Lifetime Version (K-SADS-PL). Ésta es una entrevista semiestructurada que evalúa 46 diagnósticos psiquiátricos en el presente y a lo largo de la vida. La confiabilidad interevaluador se ha reportado entre 0.66 y 1.0, dependiendo del diagnóstico. ${ }^{22}$ Todos los evaluadores fueron psiquiatras de niños y adolescentes que contaban con la capacitación requerida para la aplicación del instrumento.

El funcionamiento psicosocial se evalúo con la Escala de Evaluación Global para Niños (C-GAS), la cual se encuentra incluida en el último apartado del K-SADS-PL. Consta de una puntuación del 1 al 100, donde el mayor puntaje corresponde a un mejor funcionamiento en las áreas académica, familiar y social. ${ }^{22}$ Para la evaluación de la variable de Funcionamiento Global, se crearon tres categorías: a) sin disfunción (GAF de 71-100); b) disfunción moderada (GAF de 41-60), con interferencia en al menos un área y/o en la mayor parte de las áreas sociales; y c) disfunción grave (GAF 11-40), que incluyó deterioro grave en el funcionamiento de diversas áreas.

Así mismo, se evaluó el mejor nivel de funcionamiento actual (últimas dos semanas), en el episodio más severo en 
el pasado (EMSP), así como el mejor nivel de funcionamiento en el pasado (durante el año pasado).

\section{Procedimiento}

El protocolo fue sometido y aprobado a los comités científicos y de ética de la institución donde se llevó a cabo el estudio.

Se obtuvo el consentimiento informado de los padres y el asentimiento de los hijos. Los primeros se evaluaron con la MINI, tras lo cual se asignaron al grupo de TBP o sin TBP de la comunidad. En una segunda cita se aplicaron el K-SADS-PL y el C-GAS a sus hijos.

\section{Análisis estadístico}

Para describir las variables demográficas y clínicas se utilizaron medidas de tendencia central. A su vez, para las variables categóricas se usaron la $\chi^{2}$ y la prueba exacta de Fisher cuando el número de observaciones fue inferior a 5 . También se utilizó la $\mathrm{t}$ de Student en variables continuas para comparar porcentajes y medias entre los grupos. Se utilizaron cuadros de 2 por 2 para estimar la razón de momios. Se estableció la significancia estadística con un valor de p<0.05 a dos colas. El análisis se realizó con el programa estadístico IBM SPSS Statistics, versión 20.

\section{RESULTADOS}

\section{Características sociodemográficas}

La muestra incluyó a 62 niños y adolescentes: 31 hijos de padres con TBP y 31 hijos de padres sin TBP. En los hijos de padres con TBP, la edad promedio fue significativamente mayor que en el grupo de comparación (12.2 [DE \pm 3.55] vs. 10.1 [DE \pm 1.55], t=-2.895, p=.006). El grupo de hijos de padres con TBP alcanzó una proporción significativamente menor de participantes del sexo masculino (19 [61\%] vs. 26 [84\%], $\left.\chi^{2}=.397, \mathrm{p}=.046\right)$, mientras que la mayoría de los hijos de padres sin TBP provenía de familias biparentales (13 [42\%] vs. $\left.24[77 \%], \chi^{2}=8.11, \mathrm{p}=0.04\right) . \mathrm{Al}$ comparar si habían recibido algún tratamiento farmacológico a lo largo de la vida, no hubo diferencias significativas entre los grupos (7 [22.58\%] vs. 3 [9.7\%], p=0.116) (cuadro 1).

\section{Diagnósticos psiquiátricos en hijos de padres con trastorno bipolar versus hijos de padres sin trastorno bipolar}

Con el objetivo de reducir la probabilidad de un error tipo II para la variable trastornos psiquiátricos, en un primer análisis se decidió agrupar los trastornos en las siguientes categorías: cualquier trastorno externalizado (TDAH, trastorno negativista y desafiante [TND] y TD), cualquier trastorno afectivo (distimia, trastorno depresivo mayor y/o TBP), cualquier trastorno de ansiedad (ansiedad generalizada, ansiedad de separación, fobia social, fobia específica, trastorno obsesivo-compulsivo y estrés postraumático) y cualquier trastorno de eliminación (enuresis y/o encopresis) (cuadro 2).

Se encontró que $90 \%$ de los hijos de padres con TBP y 87\% de los hijos de padres sin TBP habían presentado algún trastorno psiquiátrico a lo largo de la vida $\left(\chi^{2}=0.161, \mathrm{p}=0.602\right)$. Los hijos de padres con TBP presentaron mayor frecuencia para cualquier trastorno externalizado que los hijos de padres sin TBP (25 [80.6\%] vs. 15 [48.4\%], $\left.\chi^{2}=7.045, \mathrm{p}=0.008\right)$. No se encontraron diferencias estadísticamente significativas entre grupos para el resto de las categorías (figura 1).

En el análisis para cada diagnóstico psiquiátrico, los hijos de padres con TBP presentaron mayor frecuencia de TDAH (22 [71\%] vs. 13 [41.9\%], $\left.\chi^{2}=5.314, \mathrm{p}=0.021\right)$ TND (16 [51.6\%] vs. 8 [25.8\%], $\chi^{2}=4.351, \mathrm{p}=.037, \mathrm{OR}=3.06, \mathrm{IC}=95 \%$, $1.05-8.93)$ y una tendencia para TD (4 [13\%] vs. $0 \mathrm{p}=0.076)$. Dos hijos de padres con TBP $(6.5 \%)$ presentaron diagnóstico de TBP no especificado. El grupo de hijos de padres sin TBP presentó mayor frecuencia de fobia social ( $\mathrm{n}=9$ [29\%] vs. $\mathrm{n}=2$ [6.5\%] vs. $\mathrm{EF}=0.043, \mathrm{p}=.020)$.

El análisis de riesgo mediante la razón de momios (R.M.) mostró que el grupo de hijos de padres con TBP tenía mayor riesgo de presentar cualquier trastorno ex-

Cuadro 1. Características sociodemográficas en hijos de padres con TBP vs. hijos de padres sin TBP

\begin{tabular}{|c|c|c|c|c|c|c|c|}
\hline \multirow[b]{2}{*}{ Características } & \multicolumn{2}{|c|}{$\begin{array}{c}\text { Hijos de padres } \\
\text { con TBP }\end{array}$} & \multicolumn{2}{|c|}{$\begin{array}{c}\text { Hijos de padres } \\
\sin \text { TBP }\end{array}$} & \multicolumn{3}{|c|}{ Análisis estadístico } \\
\hline & Media & D.E. & Media & D.E. & Prueba & $\mathrm{df}$ & $p$ \\
\hline \multirow[t]{2}{*}{ Edad (años) } & 12.2 & 3.55 & 10.1 & 1.55 & $t$ & 2.895 & 0.006 \\
\hline & $\mathbf{N}$ & $\%$ & $\mathbf{N}$ & $\%$ & & & \\
\hline Sexo masculino & 19 & 61.0 & 26 & 84.0 & $\chi^{2}$ & 0.397 & 0.046 \\
\hline Mestizos & 31 & 100.0 & 31 & 100.0 & NA & NA & NA \\
\hline Familia biparental & 13 & 42.0 & 24 & 77.0 & $\chi^{2}$ & 8.110 & 0.040 \\
\hline Historia de Abuso* & 16 & 52.0 & 9 & 9.0 & $\chi^{2}$ & 3.280 & 0.070 \\
\hline T. Farmacológico\& & 7 & 23.0 & 3 & 10.0 & $\mathrm{FE}$ & 0.170 & 0.116 \\
\hline
\end{tabular}

* Incluye cualquier categoría de abuso físico, psicológico, descuido y/o sexual alguna vez en la vida.

\& Historia de haber recibido tratamiento farmacológico alguna vez en la vida. 


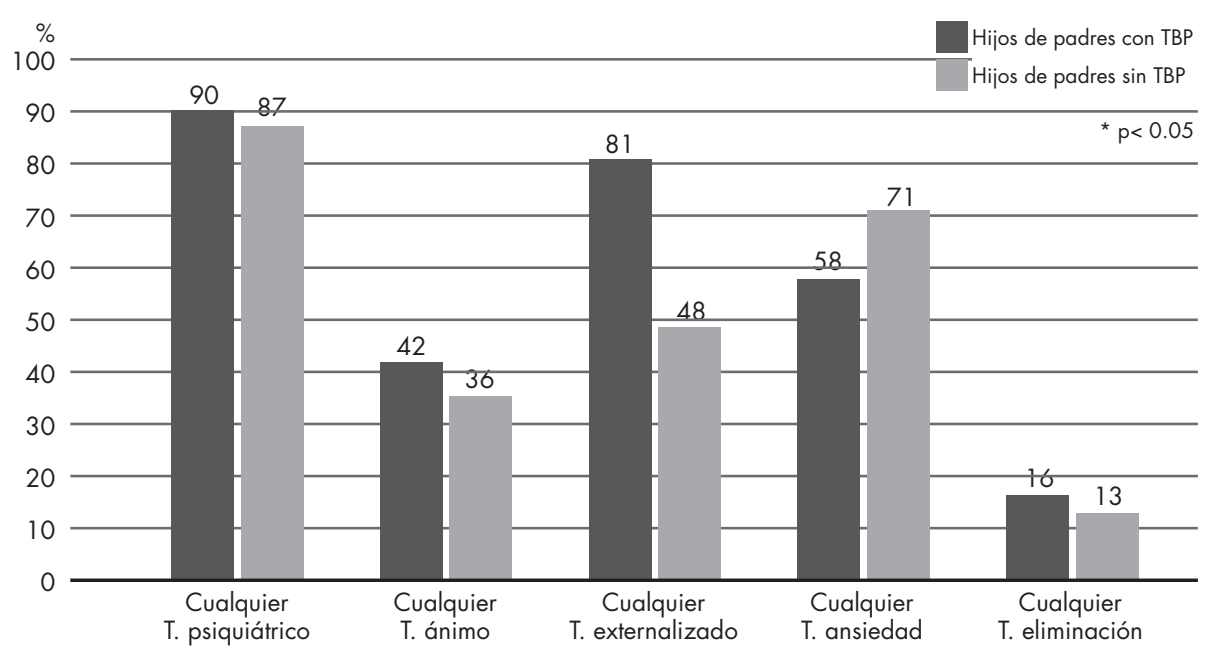

Figura 1. Comparación de categorías diagnósticas en hijos de padres con trastorno bipolar vs. hijos de padres sin TBP.

ternalizado (R.M. $=4.44$, IC 95\%, 1.43-13.84, p=.01), TDAH (R.M. $=3.38$, IC 95\%, 1.18-8.93, p=0.04) y TND (R.M.=3.07, IC $95 \%, 1.05-8.93, \mathrm{p}=.06)$.

\section{Funcionamiento global de hijos de padres con TBP versus grupo de comunidad}

El $64.5 \%$ de los hijos de padres con TBP presentaron disfunción moderada en el episodio actual en comparación con
$29 \%$ de los hijos de padres sin TBP, lo cual representó una diferencia estadísticamente significativa entre grupos (20 [64.5\%] vs. $\left.9[29 \%] \chi^{2}=.783, \mathrm{p}=.005\right)$.

\section{DISCUSIÓN}

El presente estudio comparó los trastornos psiquiátricos en hijos de padres con trastorno bipolar tratados en un hospital

Cuadro 2. Diagnósticos psiquiátricos (DSM-IV) en hijos de padres con TBP ( $n=31)$ vs. hijos de padres $\sin \operatorname{TBP}(n=31)$

\begin{tabular}{|c|c|c|c|c|c|c|c|}
\hline \multirow[b]{2}{*}{ Trastorno } & \multicolumn{2}{|c|}{$\begin{array}{c}\text { Hijos de padres } \\
\text { con TBP }\end{array}$} & \multicolumn{2}{|c|}{$\begin{array}{c}\text { Hiijos de padres } \\
\text { sin TBP }\end{array}$} & \multicolumn{3}{|c|}{ Análisis estadístico } \\
\hline & $\mathrm{N}$ & $\%$ & $\mathrm{~N}$ & $\%$ & Prueba & $\mathrm{df}$ & $\mathrm{p}$ \\
\hline TBP No especificado & 2 & 6.5 & 0 & 0.0 & $\mathrm{EF}$ & 0.492 & 0.229 \\
\hline Depresión mayor & 5 & 16.1 & 2 & 6.5 & EF & 0.425 & 0.151 \\
\hline Ansiedad generalizada & 2 & 6.5 & 6 & 19.4 & EF & .255 & 0.130 \\
\hline Ansiedad de separación & 11 & 35.5 & 9 & 29.0 & $\chi^{2}$ & .295 & 0.587 \\
\hline Fobia social* & 2 & 6.5 & 9 & 29.0 & FE & .043 & 0.020 \\
\hline Fobia específica & 11 & 32.3 & 16 & 51.6 & $\chi^{2}$ & 1.676 & 0.196 \\
\hline Obsesivo compulsivo & 1 & 3.0 & 0 & 0.0 & EF & 1.000 & 0.313 \\
\hline Estrés postraumático & 0 & 0.0 & 1 & 3.0 & EF & 1.000 & 0.313 \\
\hline TDAH* & 22 & 71.0 & 13 & 42.0 & $\chi^{2}$ & 5314 & 0.021 \\
\hline TOD* & 16 & 51.7 & 8 & 26.0 & $\chi^{2}$ & 4351 & 0.037 \\
\hline T. disocial** & 4 & 13.0 & 0 & 0.0 & $\mathrm{EF}$ & .238 & 0.076 \\
\hline Enuresis & 3 & 10.0 & 5 & 16.0 & $\mathrm{EF}$ & .707 & 0.449 \\
\hline Encopresis & 1 & 3.2 & 0 & 0.0 & $\mathrm{EF}$ & 1.000 & 0.313 \\
\hline Anorexia $\mathrm{N}$ & 2 & 6.5 & 0 & 0.0 & EF & .492 & 0.151 \\
\hline Bulimia & 1 & 3.2 & 1 & 3.2 & NA & NA & NA \\
\hline \multicolumn{8}{|l|}{ Reacciones de ajuste } \\
\hline - Con ánimo depresivo & 5 & 16.0 & 5 & 16.0 & EF & .425 & 0.151 \\
\hline - Con ansiedad** & 3 & 10.0 & 0 & 0.0 & EF & .238 & 0.076 \\
\hline
\end{tabular}


de tercer nivel de atención con hijos de padres sin TBP de comunidad. Los resultados mostraron que ambos grupos presentaron una alta tasa de psicopatología. Sin embargo, en el grupo de hijos de padres con TBP, ésta fue más del doble a la esperada para el grupo de edad $(90 \%$ de los participantes tuvo un trastorno psiquiátrico). Este hallazgo es de relevancia debido a que coloca a esta población en una posición de alto riesgo para presentar cualquier trastorno psiquiátrico a lo largo de la vida. Así también, los hijos de padres con TBP constituyeron un grupo con un mayor número de trastornos comórbidos (de tres a siete diagnósticos) y mayor disfunción, lo cual los ubica como una población diferente en términos de gravedad cuando se les compara con hijos de padres sin TBP.

Al realizar la comparación por grupo de diagnósticos, se observó que hubo una mayor frecuencia de padecimientos del espectro externalizado para los hijos de padres con TBP, quienes tuvieron 4.44 veces mayor riesgo de presentar cualquier trastorno externalizado; 3.38 y 3.07 veces mayor riesgo de presentar TDAH y TND, respectivamente. Este hallazgo es interesante debido a que, a pesar de que la muestra total de niños y adolescentes fue obtenida de la comunidad, en el presente estudio la frecuencia de trastornos externalizados en hijos de padres con TBP fue casi 15 veces más alta que la reportada en otros estudios con población general ${ }^{23} \mathrm{y}$ casi 50 veces más alta que la reportada en la encuesta nacional de adolescentes. ${ }^{24}$

Los resultados también son consistentes con otros estudios internacionales que reportan tasas elevadas de trastornos de conducta disruptiva y TDAH en los hijos de padres con TBP cuando se comparan con hijos de padres sanos ${ }^{19}$ y/o con otra psicopatología diferente al TBP.$^{14}$ La relevancia de la presencia de TDAH en población de riesgo para desarrollar TBP se ha demostrado con los seguimientos longitudinales, los cuales reportan que la asociación de TDAH con trastorno de conducta y episodios depresivos incrementa significativamente el riesgo de un viraje hacia la manía en niños y adolescentes. ${ }^{25}$ Por este motivo, algunos autores han planteado que, en poblaciones de riesgo, el TDAH puede constituir una fase prodrómica o una manifestación temprana de TBP, acorde a la etapa del desarrollo. ${ }^{16,26}$ Otra de las hipótesis propuestas es que el TBP y el TDAH comparten un sustrato biológico para la etiología de la enfermedad. ${ }^{26} \mathrm{Sin}$ embargo se requieren estudios de cohortes, genéticos y de neuroimagen que ayuden a aclarar esta asociación. Es importante que los niños y adolescentes incluidos en estudios como éste puedan tener un seguimiento.

Por otra parte, los trastornos del ánimo fueron más frecuentes en el grupo de hijos de padres con TBP; no hubo, sin embargo, diferencias con los hijos de padres sin TBP. Una probable explicación de lo anterior es que en el presente estudio la mayoría de los evaluados no había alcanzado la edad de mayor riesgo para la presencia de trastornos afectivos. ${ }^{18,23}$ Los niños que presentaron síntomas de elevación del estado de ánimo no cumplieron con el criterio de tiempo de acuerdo con el DSM-IV para confirmar el diagnóstico de TBP-I o II. No obstante, las investigaciones muestran que estos niños pueden diagnosticarse con TBP no especificado, el cual tiene un comportamiento similar a los subtipos I y II en relación con la cronicidad y disfunción. ${ }^{27}$ Algunos autores han modificado los criterios diagnósticos para TBP pediátrico tomando en consideración el número de horas en que se presentan los síntomas de elevación del estado de ánimo y durante días distintos. ${ }^{27}$ Sin embargo, esta modalidad diagnóstica incrementa la prevalencia del TBP pediátrico.

Los trastornos de ansiedad se presentaron con mayor frecuencia en los hijos de padres sin TBP. Este hallazgo estuvo probablemente relacionado con una mayor sensibilidad al reconocimiento de psicopatología por los padres al aceptar que sus hijos fueran evaluados. Por otra parte, los resultados concuerdan con estudios de muestras comunitarias mexicanas, donde los trastornos de ansiedad son los más prevalentes, pero también los menos discapacitantes, ${ }^{28}$ y finalmente un porcentaje de la muestra proveniente de la escuela fue referida por profesores, quienes pudieron haber detectado una manifestación de conducta anormal en un niño y sugerir su participación en el estudio.

Los hijos de padres con TBP tuvieron un menor nivel de funcionamiento global en el presente, con deterioro en al menos un área de acuerdo con el C-GAS. A pesar de lo anterior, la mayoría de estos niños no había recibido intervenciones terapéuticas y aquellos que sí contaron con el antecedente de tratamiento médico, recibieron el grupo de fármacos más utilizado que fue el de los estimulantes. Lo anterior sugiere que los síntomas externalizados fueron el principal motivo de disfunción.

La interpretación de los resultados del presente estudio entraña algunas limitaciones dado que el diagnóstico psiquiátrico sólo pudo evaluarse en el padre participante. No se obtuvo información sobre el otro padre biológico. Cabe mencionar también la dificultad para reclutar padres de la comunidad sin psicopatología, lo cual se ha reportado en otros estudios con diseños similares, en que 50\% de los padres de comunidad tienen al menos un diagnóstico psiquiátrico.

Aunque los evaluadores de los niños y adolescentes no fueron ciegos al diagnóstico de los padres, el uso de una entrevista semiestructurada validada en población mexicana y aplicada por psiquiatras de niños y adolescentes, capacitados y con amplia experiencia en el uso de la entrevista, así como haber contado con un grupo de comparación, fortalece los resultados obtenidos.

Según nuestro conocimiento, el presente es el segundo reporte de psicopatología en niños y adolescentes mexicanos considerados como grupos de riesgo para presentar trastorno bipolar. Dado que replica los resultados de estudios hechos en este campo en otras partes del mundo, puede servir como marco teórico para elaborar estudios subsecuentes, planes de detección y manejo de poblaciones especiales, así 
como de sustento para la implementación de programas de tamizaje en poblaciones de alto riesgo, como lo constituyen los hijos de padres con cualquier psicopatología que acuden a recibir tratamiento psiquiátrico.

\section{CONCLUSIONES}

El presente estudio reporta la psicopatología en hijos de pacientes con TBP. La elevada frecuencia de padecimientos psiquiátricos encontrada en esta población, específicamente para los trastornos externalizados, genera la necesidad de enfocar en el área clínica la atención en programas de prevención, diagnóstico y/o tratamiento oportuno para este grupo de alto riesgo.

\section{AGRADECIMIENTOS}

Los autores agradecemos y expresamos nuestro reconocimiento a todos los padres, niños y adolescentes de las familias participantes que hicieron posible este trabajo.

\section{REFERENCIAS}

1. Judd LL, Akiskal H. The prevalence and disability of bipolar spectrum disorders in the US population: re-analysis of the ECA database taking into account subthreshold cases. J Affective Disorders 2003;73:123-131.

2. Coryell W, Scheftner W, Keller M, Endicott J et al. The enduring psychosocial consequences of mania and depression. American J Psychiatry 1993;150:720-727.

3. Kessler RC, Nelson CB, McGonagle KA, Edlund MJ et al. The epidemiology of co-occurring addictive and mental disorders: implications for prevention and service utilization. American J Orthopsychiatry 1996;66:17-31.

4. Dalton EJ, Cate-Carter TD, Mundo E, Parikh SV et al. Suicide risk in bipolar patients: the role of co-morbid substance use disorders. Bipolar Disorders 2003;5:58-61.

5. Stensland MD, Zhu B, Ascher-Svanum H, Ball DE. Costs associated with attempted suicide among individuals with bipolar disorder. J Ment Health Policy 2010;13(2):87-92.

6. Leboyer M, Henry C Paillere-Martinot ML, Bellivier F. Age at onset in bipolar affective disorders: a review. Bipolar Disorder 2005;7:111-118.

7. Winokur G, Coryell W, Endicott J, Akiskal H. Further distinctions between manic-depressive illness (bipolar disorder) and primary depressive disorder (unipolar depression). American J Psychiatry 1993;150:1176-1181.

8. Henin A, Biederman J, Mick E, Dina R et al. Childhood antecedent disorders to bipolar disorder in adults: A controlled study. J Affective Disorders 2007;99:51-57.

9. Carlson GA, Bromet EJ, Driessens C, Mojtabai R et al. Age at onset, childhood psychopathology and 2 year outcome in psychotic bipolar disorder. American J Psychiatry 2002;159:307-309.

10. Lapalme M, Hodgind S, Laroche C. Children of parents with bipolar disorder: a meta-analysis of risk for mental disorders. Can J Psychiatry 1997;42:623-631.
11. Craddock N, Jones I. Genetics of bipolar disorder. J Medicine Genetics 1999;36:585-594.

12. Chang K, Steiner H, Ketter T. Psychiatric Phenomenology of Child and Adolescent Bipolar Offspring. American Academy Child Adolescent Psychiatry 2000;39:453-460.

13. Reichart C, Wals M, Hillegers M, Ormel J et al. Psychopathology in the adolescent offspring of bipolar parents. J Affective Disorders 2004;78:67-71.

14. Henin A, Biederman J, Mick E, Sachs G et al. Psychopahology in the offspring of parents with bipolar disorder: A controlled study. Biological Psychiatry 2005;58:554-561.

15. Birmaher B, Axelson D, Monk K, Kalas C et al. Lifetime psychiatric disorders in school-aged offspring of parents with bipolar disorder. The Pittsburgh bipolar offspring study. Arch Gen Psychiatry 2009;66:287-297.

16. Hirshfeld Becker D, Biederman J, Henin A, Faraone S et al. Psychopathology in the young offspring of parents with bipolar disorder: A controlled pilot study. Psychiatry Research 2006;145:155-167.

17. Duffy A, Alda M, Hajek T, Grof P. Early course of bipolar disorder in high-risk offspring: prospective study. British J Psychiatry 2009;195:457-458.

18. Duffy A, Alda M, Crawford L, Milin R, Grof P. The early manifestations of bipolar disorder: a longitudinal prospective study of the offspring of bipolar parents. Bipolar Disorder 2007;9:828-838.

19. Birmaher B, Axelson D, Goldstein B, Monk K et al. Psychiatric disorders in preschool offspring of parents with bipolar disorder: The Pittsburgh bipolar offspring study (BIOS). Am J Psychiatry 2010;167:321330.

20. Hillegers MH, Reichart CG, Wals M, Verhulst FC et al. Five-year prospective outcome of psychopathology in the adolescent offspring of bipolar parents. Bipolar Disorder 2005;7:344-350.

21. Sheehan DV, Lecrubier $Y$, Sheehan $\mathrm{KH}$, Amorim $P$ et al. The Mini International Neuropsychiatric Interview (M.I.N.I): the development and validation of a structured diagnostic psychiatric interview for DSM-IV and ICD-10. J Clin Psychiatry 1998;59(Sup 120):22-23.

22. Ulloa RE, Ortiz S, Higuera F, Nogales I et al. Interrater reliability of the Spanish version of Schedule for Affective Disorders and schizophrenia for school-age children--present and lifetime version (K-SADSPL). Actas Esp Psiquiatr 2006;34:36-40.

23. Merikangas $\mathrm{K}, \mathrm{He} \mathrm{J}$, Burstein $\mathrm{M}$, Swanson $\mathrm{S}$ et al. Lifetime prevalence of mental disorders in U.S. adolescents: results from the national comorbidity survey adolescent supplement (NCSA-A). J Am Acad Child Adolesc Psychiatry 2010;49(10):980-989.

24. Benjet C, Borges G, Medina-Mora ME, Zambrano J et al. Youth mental health in a populous city of the developing world: results from the Mexican adolescent mental health survey. J Child Psychol Psychiatry 2009;50:386-395.

25. Biederman J, Petty C, Byrne D, Wong P et al. Risk for switch from unipolar to bipolar disorder in youth with ADHD: A long term prospective controlled study. J Affective Disorders 2009;119:116-121.

26. Singh MK, Del Bello MP, Kowatch RA, Strakowski SM. Co-ocurrence of bipolar and attention-deficit hyperactivity disorders in children. Bipolar Disorder 2006;8:710-720.

27. Birmaher B, Axelson D, Goldstein B, Strober M et al. Four-year longitudinal course of children and adolescents with bipolar spectrum disorders: The course and outcome of bipolar youth (COBY) study. American J Psychiatry 2009;166:795-804.

28. Medina ME, Borges G, Lara C, Benjet C. Prevalencia de trastornos mentales y uso de servicios: Resultados de la encuesta nacional de epidemiología psiquiátrica en México. Salud Mental 2003;26(4):1-16.

Artículo sin conflicto de intereses 